\title{
Treatment of localised prostate cancer with radiation therapy: evidence versus opinion
}

\author{
Ferran Guedea • Alfredo Ramos • Ismael Herruzo • José Antonio Sánchez Calzado • Jorge Contreras • \\ Jesús Romero · Jordi Craven-Bartle · Patricia Willisch, on behalf of SEOR (Spanish Society \\ of Radiation Oncology)
}

José Luis López Torrecilla $\cdot$ Xavier Maldonado $\cdot$ Gemma Sancho $\cdot$ Almudena Zapatero, on behalf of UROONCOR (Prostate Group of SEOR)

\author{
Montserrat Ferrer · Yolanda Pardo · Pablo Fernández · Alfonso Mariño · Asunción Hervás · \\ Víctor Macías • Ana Boladeras • Ferran Ferrer, members of the Multicentric Spanish Group \\ of Clinically Localized Prostate Cancer
}

Brian J. Davis, Chairman of the American Brachytherapy Society

On the rare occasions when two different medical specialties both offer good therapeutic outcomes for the same disease, the result is usually a debate. When this debate involves enthusiastic individuals who love their professions, it can easily become quite passionate - not because of any partisan interests, but rather because both sides are convinced that their treatment is the best for the patient. In the treatment of localised prostate cancer, radiation oncologists and urologists both face the same uncertainties when informing patients of the advantages and disadvantages of the various existing treatment options [1,2]. In this context, Actas Urológicas Españolas, the journal of the Spanish Urological Association, has recently published an editorial [3] titled "Critical review of use of radiation as initial treatment for localised prostate cancer". In the course of any debate, it is useful to analyse the strengths and weaknesses of each therapeutic modality. It is this process of constructive critical analysis that leads to continuous improvement. However, emphasising a single aspect of treatment while ignoring the bigger picture is a questionable tactic. This is the difference between a systematic review of the clinical evidence [1] and opinion based on a critical review of selected publications. Opinion certainly has value, especially when it comes from a highly qualified specialist, as it can serve to guide the management of a concrete clinical situation in a specific context or centre. However, in contrast to evidence-based medicine, opinion -despite its allure at

F. Guedea (ه)

Department of Radiation Oncology

Institut Català d'Oncologia

Gran vía, s/n - km 2,7

ES-08907 L'Hospitalet de Llobregat, Barcelona, Spain

e-mail: guedea@iconcologia.net times- cannot and should not establish or change the therapeutic indication, cannot be applied universally, and most definitely should not prevail over evidence.

As physicians, we all surely agree that our main objective is to offer patients the best treatment available - in other words, treatment that offers the highest cure rate with minimal functional repercussions. It is difficult to establish the superiority of one treatment over another when the consensus is that there is insufficient data to do so [1] and while a wide range of strategies -including watchful waiting- are still under evaluation. Nor is there a clear consensus about how to evaluate cancer control in these treatments: biochemical relapse-free survival, metastasis-free survival, positive biopsies versus pathological changes due to irradiation, etc. All these parameters are too partial to select only one. However, there is general agreement that the primary objective of all cancer treatments is to improve overall and tumor-specific survival; the other variables (such as biochemical control) are only surrogates for the early assessment of efficacy in a long-term disease. Indeed, sufficient evidence exists, as many prestigious authors acknowledge, to indicate that both external-beam radiotherapy (EBRT) and brachytherapy are techniques whose results are equivalent to radical prostatectomy [4-6].

Prostate-specific antigen (PSA) levels should not be used to compare the efficacy of radical prostatectomy versus radiotherapy, as these values are obviously conditioned by the presence or not of the prostate gland. The response of both cancerous and healthy prostatic tissue to radiation is known and predictable and follows well-known radiobiologic models that have been validated and accepted by the medical community. The most commonly used criteria to determine biochemical relapse is the American Society for Therapeutic Radiology and Oncology (ASTRO) consensus, which was published in 2006 and is known as the "Phoenix 
criteria". Relapse is considered to occur at nadir $+2 \mathrm{ng} / \mathrm{ml}$ [7]. This relapse criterion was not selected arbitrarily, but rather because it has a high sensitivity and specificity for future clinical events. The Phoenix criteria can be applied to brachytherapy patients, it is a robust measure in cases of postimplant PSA bounces and is valid for patients who receive neoadjuvant hormonotherapy.

Current external radiotherapy techniques (3D conformal radiotherapy with dose escalation), particularly image-guided intensity-modulated radiation therapy (IMRT) and brachytherapy, offer excellent rates of biochemical and local control, as well as excellent cause-specific survival in patients with localised prostate cancer. Numerous centres of excellence (Cleveland Clinic Foundation, Memorial Sloan Kettering Cancer Center, University of California San Francisco (UCSF), Massachusetts General Hospital, among others) have reported 5-year biochemical control rates ranging from $85 \%$ to $98 \%$, both with EBRT and brachytherapy [8]. The Urology Department at Mount Sinai Hospital recently published results for their series of patients treated with brachytherapy, reporting local control rates of $96.9 \%$ for implants with a biologically equivalent dose (BED) >200 Gy [9]. In Spain, the Grupo de Investigación Clínica en Oncología Radioterápica (GICOR) trial published in 2005 showed a biochemical relapse-free survival rate of $80 \%$ in patients treated with a mean of $72 \mathrm{~Gy}$ of 3D-EBRT [10].

The potential for developing radiation-induced cancer is small but real. However, the results of studies carried out to investigate this risk are contradictory. A recent study estimated that the hazard ratio of developing a rectal tumour at 120 months was 2.2 in patients treated with radiotherapy compared with the general population [11]. In contrast, a Canadian study evaluated all prostate cancer cases treated in British Columbia from 1984 to 2000 and found no significant difference between observed and expected second cancer rates, whether treatment included radiotherapy or not [12]. It is well known that radiotherapy is more effective at killing cancer cells than creating them. The data on prostate cancer and second malignancies are not conclusive and therefore do not provide a sufficient scientific basis for refusing to offer patients a curative treatment option. The difficulty lies in ascertaining whether a patient with prostate cancer has the same risk of developing a second tumour as the rest of the population, regardless of treatment modality. It is clearly the responsibility of the radiation oncologist to minimise this risk as much as possible by improving treatment planning and administration. However, because radiation oncologists are well aware of this risk, they are highly sensitive to it and therefore take a proactive approach to even the smallest signs suggestive of a second neoplasia. As a result, any second tumour is usually detected in the early stages.

Results of the first longitudinal studies with pretreatment evaluation [13-16] designed to assess the impact of treatment side effects on quality of life in patients with localised prostate cancer have recently been published, including a study performed by the Multicentric Spanish Group of Clinically Localized Prostate Cancer [14]. All these studies agree that there are important differences in terms of side effects among treatments: radical prostatectomy (with or without nerve conservation) causes the most urinary incontinence and sexual dysfunction, whereas conformal EBRT presents the largest adverse effects on bowel function. The most notable effects of brachytherapy are irritative-obstructive urinary symptoms. Although brachytherapy clearly has less impact on sexual function than surgery, some differences between the studies were seen in this regard.

Theoretically, new surgical techniques, such as laparoscopic or robotic surgery, could reduce the side effects associated with prostatectomy. However, studies published to date present certain limitations, and the results are not consistent. The systematic review of studies of robotic, laparoscopic and radical retropubic prostatectomy [17] cited in the aforementioned editorial concluded that the perioperative results of laparoscopic and robotic surgery (transfusions, catheterisation time, days hospitalised and complications of the intervention) are more favourable than older techniques. However, that same review found that most published studies did not use standardised and validated measures to evaluate continence and potency.

It is clearly not possible to compare morbidity between surgery and radiotherapy due to the inherent differences in these procedures. Quality control programmes should be reviewed periodically to minimise the risk of error. In the case of radiation oncology, these programmes are regulated by Royal Decree (national law), and all processes associated with treatment, therapeutic efficacy and follow-up for potential morbidity are subject to these regulations [18]. Because of this rigorous self-assessment, procedures vary little from one centre to another and are not subject to individual factors. In order to assess and subsequently register radiotherapy-associated morbidity, the European Organization for Research and Treatment of Cancer (EORTC) and the Radiation Therapy Oncology Group (RTOG) have designed and validated acute and chronic toxicity scales. These standardised scales are used by radiation oncologists throughout the world. In the most recent series of patients treated with IMRT (dose $81 \mathrm{~Gy}$ ), only $4 \%$ of patients experience late rectal toxicity of grade 2 or higher. For urinary toxicity, the percentage (9\%) was higher, mainly due to an increase in urinary frequency. Grade 3 or 4 toxicity is uncommon nowadays [19]. This standardisation is not found in series of prostatectomy patients. The experience of Memorial Sloan Kettering Cancer Center was recently published [20]. In that study, the authors criticised the lack of institutional standardisation in reporting complications. They reviewed 4,592 prostatectomies and found medical and surgical complication rates of $10.2 \%$ and $20.1 \%$, respectively; complications were severe in $2.3 \%$ (medical) and $6.6 \%$ (surgical) of cases. Each institution should contrast their own results with these data, both to activate 
mechanisms for improvement and to inform patients of the real risk of complications.

Selection of a therapeutic approach for localised prostate cancer should take into account additional factors: clinical stage, experience of the centre, expected quality of life and psychosocial aspects of the individual. This implies that the information we give to patients must, of necessity, be complex. Patients must be actively involved in selecting the treatment modality, and this requires that we provide reliable information based on quality scientific evidence. If we are to properly interpret the results of series of patients or data from a hospital or cancer registry, it is important that patients be grouped by internationally accepted risk groups. As no validated molecular and genetic factors are available at present, definition of these risk groups is based on tumour spread, initial PSA, and Gleason score. Age is not considered in staging. These variables identify, roughly, several different phenotypes with a well-differentiated natural history. Any attempt to compare treatments for prostate cancer without first staging patients and then sorting them by risk groups is scientifically unacceptable.

With the spectacular growth in our understanding of this disease and the introduction of new technology in recent years, the weak points of EBRT, brachytherapy, and radical prostatectomy -i.e., morbidity, side effects and the consequent impact on quality of life- have shown similar improvements in all three techniques. Indeed, progress has been so great that current morbidity and quality of life data provide positive arguments to support the selection of any of these therapeutic modalities. Dose escalation improves biochemical control in radiotherapy. At present, most radiotherapy centres in Spain are equipped with the latest technology, and it is expected that because of this, therapeutic indices will improve over time. Given these excellent results, the argument that radical prostatectomy should prevail over EBRT or brachytherapy is inconsistent; it is simply unacceptable to exclude a treatment whose efficacy (measured by biochemical control) ranges from 80-90\% merely to be sure that it is available for a possible future salvage treatment in case of recurrence after prostatectomy.

Radiation oncologists and urologists both follow the guidelines published by their respective national and international societies $[21,22]$. These guidelines are developed by internationally recognised groups of experts and are based on a review of the literature classified by levels of evidence. These documents are intensely debated by leaders of the respective societies in order to reach consensus. EBRT and brachytherapy, as well as radical prostatectomy, are all perfectly valid treatment modalities for patients with localised prostate cancer. The only way to continue improving our understanding of the benefits and adverse effects of each treatment modality is through research. Randomised clinical trials similar to the English study Prostate Testing for Cancer and Treatment (PROTECT) [23] are needed to find a response to current uncertainties.

\section{References}

1. Wilt TJ, MacDonald R, Rutks I et al (2008) Systematic review: comparative effectiveness and harms of treatments for clinically localized prostate cancer. Ann Intern Med 148:435-448

2. T. Parker-Pope, "A Review of Prostate Cancer Leaves Men in a Muddle," New York Times, 20 February, 2008

3. Esquena S, Sánchez-Martín FM, Palou Redorta Jet al (2010) Critical review of use of radiation as initial treatment for localized prostate cancer. Actas Urol Esp 34:6-9

4. Thompson I, Thrasher JB, Aus G et al (2007) Guideline for the management of clinically localized prostate cancer: 2007 update. J Urol 177: 2106

5. Zelefsky MJ, Reuter VE, Fuks Z (2008) Influence of local tumor control on distant metastases and cancer related mortality after external beam radiotherapy for prostate cancer. J Urol 179:1368-1373

6. Stone NN, Potters L, Davis BJ et al (2009) Multicenter analysis of effect of high biologic effective dose on biochemical failure and survival outcomes in patients with Gleason score 7-10 prostate cancer treated with permanent prostate brachytherapy. Int J Radiat Oncol Biol Phys 73:341-346

7. Roach M, Hanks G, Thames H et al (2006) Defining biochemical failure following radiotherapy with or without hormonal therapy in men with clinically localized prostate cancer: Recommendations of the RTOG-ASTRO Phoenix Consensus Conference. Int J Radiat Oncol Biol Phys 65: 965-974

8. Zelefsky M, Yamada Y, Fuks Z et al (2008) Longterm results of conformal radiotherapy for prostate cancer: impact of dose escalation on biochemical tumor control and distant metastases-free survival outcomes. Int J Radiat Oncol Biol Phys 71: $1028-1033$

9. Stone N, Stock RG, Cesaretti JA, Unger P (2010) Local control following permanent prostate brachytherapy: effect of high biologically effective dose on biopsy results and oncologic outcomes. Int J Radiat Oncol Biol Phys 76:355-360

10. Zapatero A, Valcarcel F, Calvo F et al. Riskadapted androgen deprivation and escalated threedimensional conformal radiotherapy for prostate cancer: does radiation dose influence outcome of patients treated with adjuvant androgen deprivation? A GICOR Study. J Clin Oncol 6561-6568

11. Bhojani N, Capitanio U, Suardi N et al (2010) The rate of secondary malignancies after radical prostatectomy versus external beam radiation therapy for localized prostate cancer: a population-based study on 17,845 patients. Int J Radiat Oncol Biol Phys 76:342-348

12. Pickles T, Phillips N (2002) The risk of second malignancy in men with prostate cancer treated with or without radiation in British Columbia, 1984-2000. Radiother Oncol 65:145-451 13. Sanda MG, Dunn RL, Michalski J et al (2008) Quality of life and satisfaction with outcome among prostate-cancer survivors. N Engl J Med 358:1250-1261

14. Ferrer M, Suarez JF, Guedea F et al (2008) Healthrelated quality of life 2 years after treatment with radical prostatectomy, prostate brachytherapy, or external beam radiotherapy in patients with clinically localized prostate cancer. Int J Radiat Oncol Biol Phys 72:421-432

15. Korfage IJ et al (2005) Five-year follow-up of health-related quality of life after primary treat- ment of localized prostate cancer. Int J Cancer 116:291-296

16. Chen RC, Clark JA, Talcott JA (2009) Individualizing quality-of-life outcomes reporting: how localized prostate cancer treatments affect patients with different levels of baseline urinary, bowel, and sexual function. J Clin Oncol 27:3916-3922

17. Ficarra V, Novara G, Artibani W et al (2009) Retropubic, laparoscopic, and robot-assisted radical prostatectomy: a systematic review and cumulative analysis of comparative studies. Eur Urol 55: 1037-1063

18. Real Decreto $1566 / 1998$, de 17 de julio, por el que se establecen los criterios de calidad en radioterapia. Ministerio de Sanidad y Consumo. B.O.E. núm. 206 del 28-08-1998, 29383-29394

19. Zelefsky M, Fuks Z, Hunt M et al (202) High-dose intensity modulated radiation therapy for prostate cancer: early toxicity and biochemical outcome in 772 patients. Int J Radiat Oncol Biol Phys 53:1111-1116

20. Rabbani F, Herran L, Pinochet R et al (2010) Comprehensive standarized report of complications of retropubic and laparoscopic radical prostatectomy. Eur Urol 57:371-386

21. Heidenreich M, Aus G, Bolla M et al. EAU guidelines on prostate cancer. Eur Eurol 53:68-80

22. National Comprehensive Cancer Network $(\mathrm{NCCN})$ : NCCN Clinical Practice Guidelines in Oncology, available at http://www.nccn.org/professionals/physician_gls/f_guidelines.asp\#site

23. Donovan J, Mills N, Smith M et al (2002) Quality improvement report: Improving design and conduct of randomised trials by embedding them in qualitative research: PROTECT (Prostate Testing for Cancer and Treatment) study. Commentary: presenting unbiased information to patients can be difficult. BMJ 325 (7367):766-770 